\title{
Basic Solutions of Three Dimensional Elasticity
}

\author{
WX Zhang ${ }^{1, *}$ \\ ${ }^{1}$ Nantong Polytechic College, Nantong, Jiangsu, 226002, China
}

\begin{abstract}
Elastic calculation method is an important research content of computational mechanics. The problems of elasticity include basic equations and boundary conditions. Therefore, the final solution consists of the general solutions of the basic equations and the special solutions satisfying the boundary conditions. Numerical method is often used in practical calculation, but the analytical solution is also an important subject for researchers. In this paper, the basic solution of three-dimensional elastic materials is given by theoretical derivation.
\end{abstract}

\section{Introduction}

Elasticity mainly studies the deformation and internal force of elastic objects under the action of external forces and other external factors [1-3]. Saint Venant's principle is the most commonly used method of elasticity, and its correctness has been fully verified. According to Saint Venant's principle, the boundary conditions can be replaced by equivalent loads, which makes the problem greatly simplified. However, the Saint Venant solution can only satisfy the average boundary conditions, ignoring the local effect, so it is an approximate solution. In many practical problems, researchers often ignore the local effect solution and only care about the overall effect of load on materials and structures. However, for some special cases, such as stress concentration caused by temperature or displacement constraints, the stress is not uniformly distributed, and the local solution plays a key role [4-6].

$$
\begin{aligned}
& \bar{u}=M_{1 j}\left(C_{j} J_{n-1}+C_{j+3} Y_{n-1}\right)+N_{1 j}\left(C_{j} J_{n}+C_{j+3} Y_{n}\right) \\
& \bar{v}=N_{2 j}\left(C_{j} J_{n}+C_{j+3} Y_{n}\right)+K_{2 j}\left(C_{j} J_{n+1}+C_{j+3} Y_{n+1}\right) \\
& \bar{w}=N_{3 j}\left(C_{j} J_{n}+C_{j+3} Y_{n}\right)+K_{3 j}\left(C_{j} J_{n+1}+C_{j+3} Y_{n+1}\right)
\end{aligned}
$$

and

$$
\begin{aligned}
& \bar{p}_{1}=N_{4 j}\left(C_{j} J_{n}+C_{j+3} Y_{n}\right)+K_{4 j}\left(C_{j} J_{n+1}+C_{j+3} Y_{n+1}\right) \\
& \bar{p}_{2}=N_{5 j}\left(C_{j} J_{n}+C_{j+3} Y_{n}\right)+K_{5 j}\left(C_{j} J_{n+1}+C_{j+3} Y_{n+1}\right) \\
& \bar{p}_{3}=M_{6 j}\left(C_{j} J_{n-1}+C_{j+3} Y_{n-1}\right)+N_{6 j}\left(C_{j} J_{n}+C_{j+3} Y_{n}\right)
\end{aligned}
$$

Respectively. Using the generalized variable method, we get

\footnotetext{
"Corresponding author's e-mail: Wxzhang697608@sina.com
} 


$$
\left\{\begin{array}{l}
a_{4} \partial_{r} \bar{u}+2 a_{1} G^{*}\left(\partial_{\theta} \bar{v}+\bar{u}\right)+a_{1} \bar{p}_{3}=\bar{\sigma}_{r}^{0}(\theta, z) \\
\partial_{r} \bar{v}+\partial_{\theta} \bar{u}-\bar{v}=a_{3} \bar{\tau}_{r \theta}^{0}(\theta, z) \\
\bar{p}_{1}=\bar{\tau}_{r z}^{0}(\theta, z)
\end{array} \quad(r=a, b)\right.
$$

and

$$
\begin{aligned}
& \left(-\mu J_{0}+2 J_{1} / r\right)\left(h_{3}+h_{4}\right)+\left(-\mu Y_{0}+2 Y_{1} / r\right)=0 \\
& 2 a_{7} \mu J_{1} h_{1}+2 a_{7} \mu Y_{1} h_{2}+\left(J_{1}-2 a_{7} \mu r J_{0}\right)\left(h_{3}-h_{4}\right)=0 \\
& 2 a_{7}\left(\mu^{2} J_{0}-\mu J_{1} / r\right) h_{1}-2 a_{7}\left(-\mu^{2} Y_{0}+\mu Y_{1} / r\right) h_{2}=0
\end{aligned}
$$

The solution can be described as follows by Bessel function:

$$
\boldsymbol{\eta}=\left[\begin{array}{cc}
\left(-a_{4} s_{1} \alpha_{13}+a_{2} \alpha_{43}\right) \sin \left(\mu a s_{1}\right) & \left(-a_{4} s_{2} \alpha_{14}+a_{2} \alpha_{44}\right) \sin \left(\mu a s_{2}\right) \\
\alpha_{33} \cos \left(\mu a s_{1}\right) & \alpha_{34} \cos \left(\mu a s_{2}\right)
\end{array}\right]
$$

The solution of the stretching problem is

$$
\begin{aligned}
& \bar{u}^{(0)}=2 \mu a_{8} r J_{0}(\mu r)-\left[1+2 \mu a_{8} M\right] J_{1}(\mu r) \\
& \bar{w}^{(0)}=\left(2 \mu a_{8} M-1\right) J_{0}(\mu r)+2 \mu a_{8} r J_{1}(\mu r) \\
& \bar{p}_{1}^{(0)}=4 \mu^{2} a_{8} r G^{*}\left[r J_{0}(\mu r)-M J_{1}(\mu r)\right] \\
& \bar{p}_{3}^{(0)}=4 \mu^{2} a_{8} r G^{*}\left[r(\mu M-2) J_{0}(\mu r)-\mu r J_{1}(\mu r)\right] \\
& \bar{v}^{(0)}=\bar{p}_{2}^{(0)}=0
\end{aligned}
$$

Numerical example

All eigenvalues and solutions can be obtained by numerical calculation. The first five displacement components and stress components are shown in Fig. 1-3, respectively. Obviously, these eigensolutions have local characteristics and must be considered in the study of stress concentration and deformation concentration.

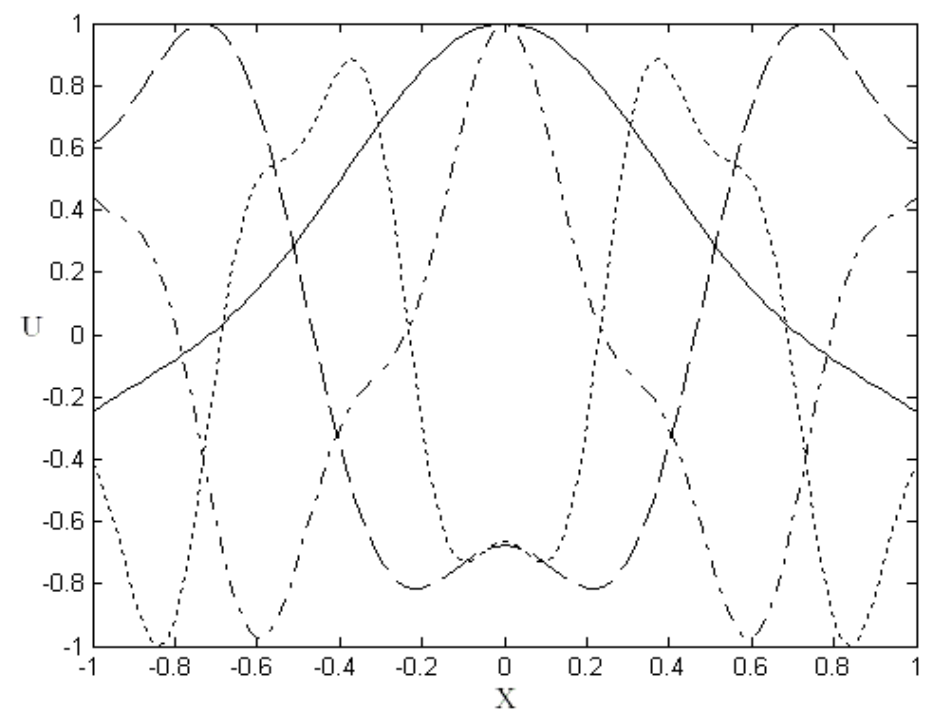

Figure 1. Component $u$ 


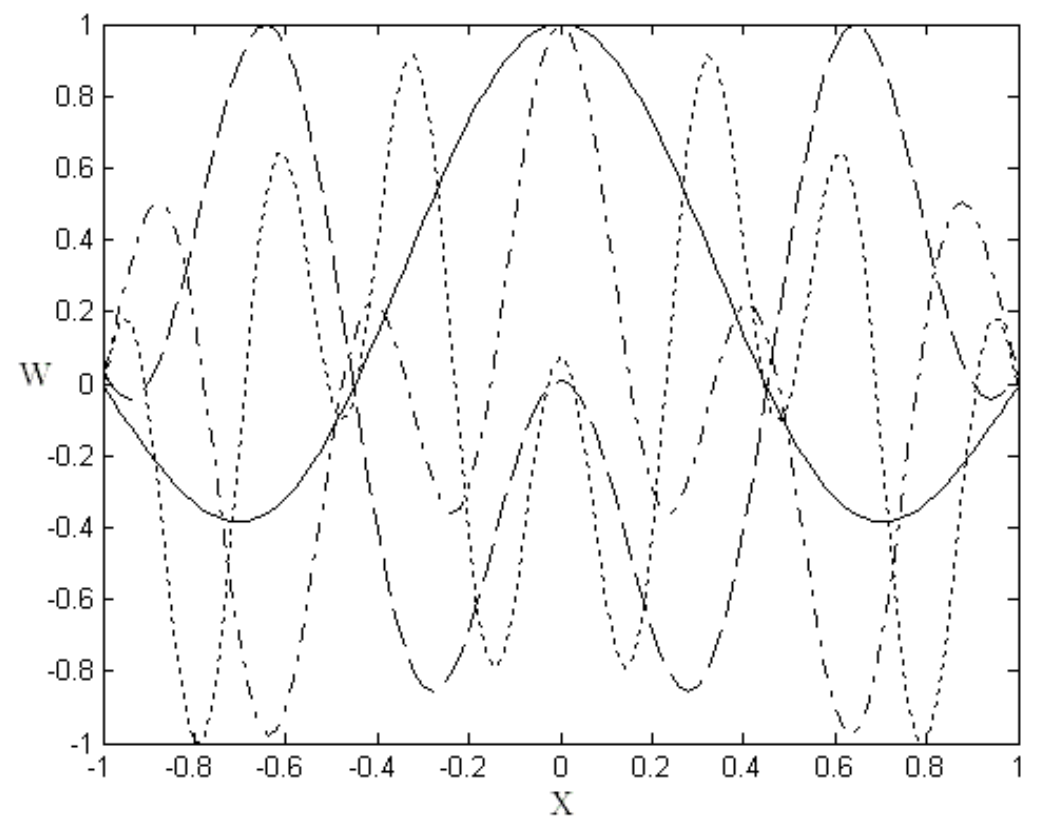

Figure 2. Component w

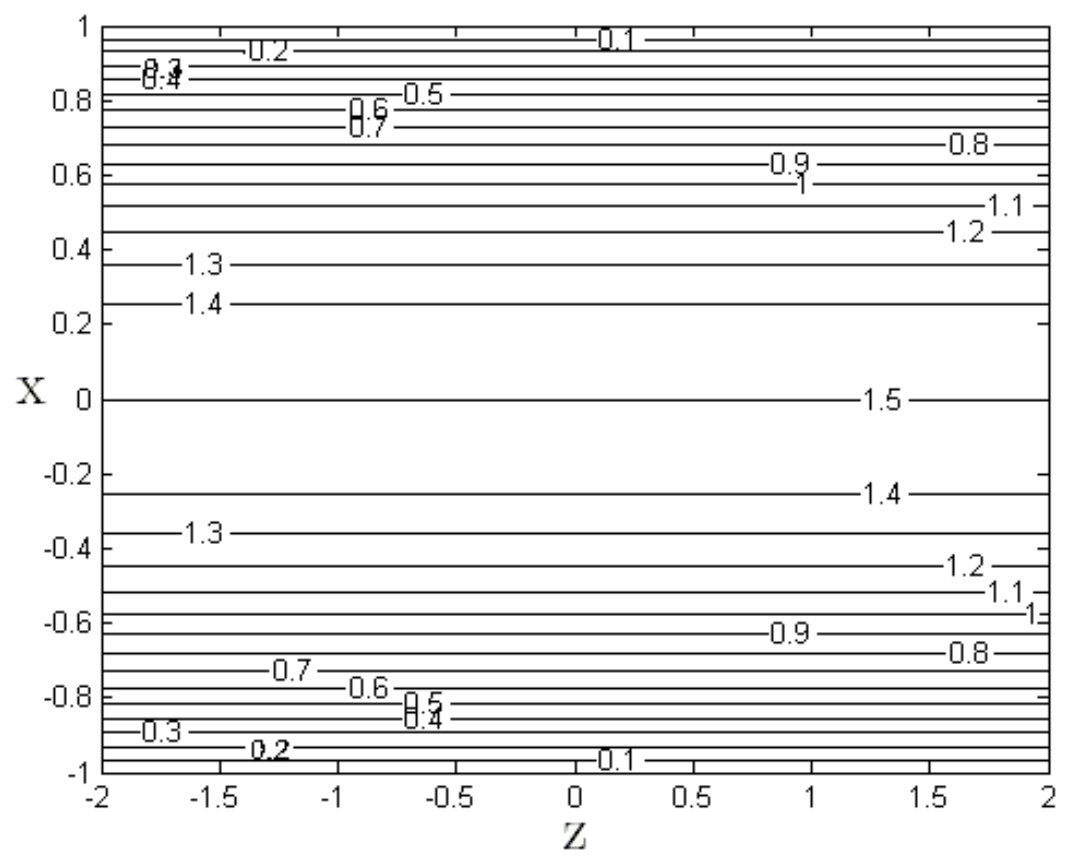

Figure 3. Stress distribution

\section{Conclusion}

Using the dual soulutions, all eigenvalues and eigensolutions can be obtained. These eigensolutions include Saint Venant's solution to describe global deformation and non-zero eigensolutions are important bases to describe local deformation.

\section{Reference}

1. Faghidian, S.A. (2016) Unified formulation of the stress field of saint-Venant's flexure problem for symmetric cross-sections. Int. J. Mech. Sci., 111-112: 65-72.

2. Pipkin, A.C., Rogers, T.G. (1968) A nonlinear integral representation for viscoelastic behavior. J. Mech. Phys. Solids, 16: 59-72. 
3. Beom, H.G., Atluri, S.N. (1996) Near-tip fifields and intensity factors for interfacial cracks in dissimilar anisotropic piezoelectric media. Int. J. Fract., 75: 163-183.

4. Perzyna, P. (1963) The constitutive equations for rate sensitive plastic models. Quart. Appl. Math., 20: 321-332.

5. Argyris, J.H., Doltsinis, I.S.T., William, K.J. (1979) New developments in the inelastic analysis of quasistatic and dynamic problems. Int. J. Numer. Meth. Eng., 14: 1813-1850.

6. Barbero, E.J., Luciano, R. (1995) Micromechanical formulas for the relaxation tensor of linear viscoelastic composites with transversely isotropic fibers. Int. J. Solids Struct., 32: 1859-1872. 